\title{
Optimización en la técnica de medida de la presión arterial pulmonar por ecocardiografía Doppler con el uso de contraste en pacientes con enfermedad pulmonar obstructiva crónica
}

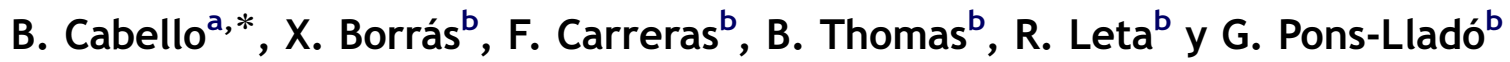 \\ ${ }^{a}$ Servicio de Medicina Intensiva, Hospital de Sant Pau, Barcelona, España \\ ' Unidad de Imagen Cardiaca, Hospital de Sant Pau, Barcelona, España
}

Recibido el 24 de marzo de 2010; aceptado el 8 de mayo de 2010

Disponible en Internet el 2 de julio de 2010

\author{
PALABRAS CLAVE \\ Presión sistólica de \\ arteria pulmonar; \\ Regurgitación \\ tricuspídea; \\ Contraste \\ ecocardiográfico; \\ Enfermedad pulmonar \\ obstructiva crónica
}

\begin{abstract}
Resumen
Objetivo: Evaluar el beneficio del contraste en la ecocardiografía para medir la presión de arteria pulmonar sistólica (PAPs).

Diseño: Estándar de referencia (ecocardiografía) comparada con contraste.

Ambito: Gabinete de ecocardiografia.

Pacientes: Ambulatorio con enfermedad pulmonar obstructiva crónica (EPOC).

Intervención: La señal de la regurgitación tricuspidea (RT) por Doppler fue evaluada antes y después de administrar contraste intravenoso (Levovist ${ }^{\mathbb{R}}$ ). Se definió una escala de 4 patrones de señal de RT: $0=$ ausencia de regurgitación; $1=$ señal protosistólica que no permite la medida de la velocidad pico; 2 =señal de intensidad no homogénea pero que permite la medida de la velocidad pico y $3=$ señal uniforme y pansistólica. El valor de la PAPs fue estimado añadiendo $10 \mathrm{mmHg}$ al gradiente transtricuspideo.

Resultados: La PAPs fue calculada solo en 20 (49\%) pacientes antes del contraste. Diecisiete pacientes fueron clasificados en el patrón 2 y 3 en el patrón 3 de la señal de la RT. Tras la administración de contraste 41 (95\%) pacientes mostraron señal de RT. Dos fueron clasificados en el patrón 1, 11 con el patrón 2 y 28 con el 3 . En los 20 pacientes en los que la PAPs fue estimada antes del contraste se observó aumento de su valor, $44 \pm 10 \mathrm{mmHg}$ vs. $56 \pm 15 \mathrm{mmHg}(\mathrm{p}<0,01)$.

Conclusión: El uso de un agente de contraste intravenoso en la ecocardiografía Doppler aumenta el número de pacientes EPOC en los que la PAPs puede ser estimada de manera no invasiva y podría evitar una infraestimación del valor de la PAPs.

(C) 2010 Elsevier España, S.L. y SEMICYUC. Todos los derechos reservados.
\end{abstract}

\footnotetext{
*Autor para correspondencia.

Correo electrónico: furones@hotmail.com (B. Cabello).
} 


\section{KEYWORDS}

Pulmonary artery systolic pressure; Tricuspid regurgitation; Contrast echocardiography; Chronic obstructive pulmonary disease

\section{Introducción}

La enfermedad pulmonar obstructiva crónica (EPOC) puede ocasionar hipertensión pulmonar y cor pulmonale ${ }^{1}$. La hipertensión pulmonar ligera o moderada puede ocurrir en la fase final de la evolución del EPOC y es secundaria a la vasoconstricción de pequeñas arterias por hipoxemia. El desarrollo de la hipertensión pulmonar en el paciente EPOC puede ocurrir incluso en pacientes con hipoxemia moderada $^{2}$ y conlleva un pronóstico infausto ${ }^{3}$.

Oswald-Mammosser et al mostraron que el valor medio de la presión de arteria pulmonar (PAP) y la edad eran los mejores factores predictivos de mortalidad en pacientes con EPOC severa que requerían oxigenoterapia ${ }^{4}$. La oxigenoterapia es actualmente el único tratamiento que reduce la mortalidad en pacientes con EPOC con hipoxemia severa en reposo $^{5,6}$ y reducciones en el valor de la PAPs han sido observados en pacientes EPOC que reciben este tratamiento ${ }^{7}$. Ha sido demostrado que el valor de la PAPs es un factor independiente predictor de hospitalización en pacientes con exacerbaciones agudas de la EPOC ${ }^{2}$.

La naturaleza invasiva de la cateterización del corazón derecho contraindica su uso rutinario para la evaluación de la PAPs en pacientes EPOC. Una estimación no invasiva puede realizarse con la ecocardiografia Doppler utlizando el valor de la velocidad máxima de la regurgitación tricuspidea (RT) para estimar el valor de la PAPs. ${ }^{8}$. Una señal interpretable del Doppler es obviamente el requisito indispensable para una estimación precisa.

El uso de la ecocardiografía Doppler en pacientes EPOC requiere asimismo una serie de condiciones únicas para el ecocardiografista secundarios a la particular morfología de la caja torácica y la hiperinsuflación pulmonar. La óptima angulación del transductor puede ser dificultosa en este contexto obteniéndose por tanto una débil señal. Un jet de RT fue posible detectarlo en solo el $30 \%$ de pacientes EPOC en un estudio multicéntrico ${ }^{8}$. De forma similar Boussuges et al no pudieron obtener una señal interpretable de ecocardiografía en 18 de 52 pacientes EPOC, lo que subraya los límites de los ultrasonidos en este subgrupo de pacientes ${ }^{9}$.

Dada la gran prevalencia de pacientes EPOC, la relevancia del valor de la PAPs y las dificultades técnicas de la ecocardiografía en este subgrupo de pacientes, decidimos utilizar un ecocontraste intravenoso basado en la galactosa (Levovist ${ }^{\mathbb{R}}$ ) para intensificar la señal de RT para estimar el valor de la PAPs en pacientes EPOC. Estudios previos han demostrado el beneficio del uso de contraste para el estudio de la regurgitación tricúspidea y medición de la presión de arteria pulmonar ${ }^{10-12}$. El suero salino agitado ha demostrado aumentar la intensidad de la RT en sujetos sanos y en pacientes EPOC ${ }^{13,14}$ pero el beneficio de la utilización de un contraste basado en galactosa en pacientes EPOC nunca ha sido estudiado previamente.

Nuestra hipótesis es que en pacientes estables EPOC el uso de un contraste ecocardiográfico intravenoso puede incrementar el número de pacientes en los que la PAPs puede ser estimada de manera no invasiva.

El objetivo del estudio es utilizar el contraste intravenoso para facilitar la medida de la PAPs en pacientes EPOC, ya que el cálculo de este valor está seriamente dificultado por las características de la caja torácica y la mala ventana acústica y su conocimiento es extremadamente importante 
ya que puede implicar modificaciones en el tratamiento del paciente y conocer el estadio de su enfermedad.

\section{Pacientes y métodos}

Este estudio fue aprobado por el Comité Ético del Hospital Sant Pau. Todos los pacientes o sus familiares fueron informados y dieron por escrito su consentimiento antes de ser incluidos en el estudio.

Cuarenta y dos pacientes EPOC descompensados fueron consecutivamente remitidos desde el departamento de neumología al laboratorio de ecocardiografía para evaluación de la PAPs. El diagnóstico de EPOC estuvo basado en la historia clínica, en la exploración física, en la radiografía, en las exploraciones funcionales y en la gasometría arterial. Ningún paciente presentaba insuficiencia cardiaca, definida como fracción de eyección de menos del $50 \%$ o historia clínica de insuficiencia cardiaca congestiva.

El estudio ecocardiográfico fue realizado con un Sonos 5.500 imager (Hewlett Packard; Andover, Mass., EE.UU.) y con una sonda S4 que funciona con tecnología de fusión de frecuencia. Todos los estudios fueron realizados por ecocardiografistas que tenían al menos 14 años de experiencia en la realización de ecocardiografías y que realizaban al menos 10 exámenes diarios. La señal de regurgitación tricuspidea fue obtenida con el Doppler continuo desde el plano apical 4 cámaras. En nuestra experiencia y en la de otros autores ${ }^{8,13,14}$ la estimación de la misma señal desde el ángulo subcostal es inferior al ángulo 4 cámaras ya que la sonda no está en paralelo con la señal de RT. La modalidad de ecocardiografía por armónico fue utilizada para todos los pacientes.

La calidad de la señal de RT fue clasificada en una escala cualitativa usando la siguiente escala: $0=$ ausencia de regurgitación; 1 =señal protosistólica que no permite la medida de la velocidad pico (fig. 1); $2=$ señal de intensidad no homogénea pero que permite la medida de la velocidad pico (fig. 2), y $3=$ señal uniforme y pansistólica (fig. 3) ${ }^{15}$. En los pacientes con los patrones 2 y 3 , el valor de la PAPs fue calculado por la señal de regurgitación tricuspidea La ecuación de Bernoulli, simplificada por Hatle and

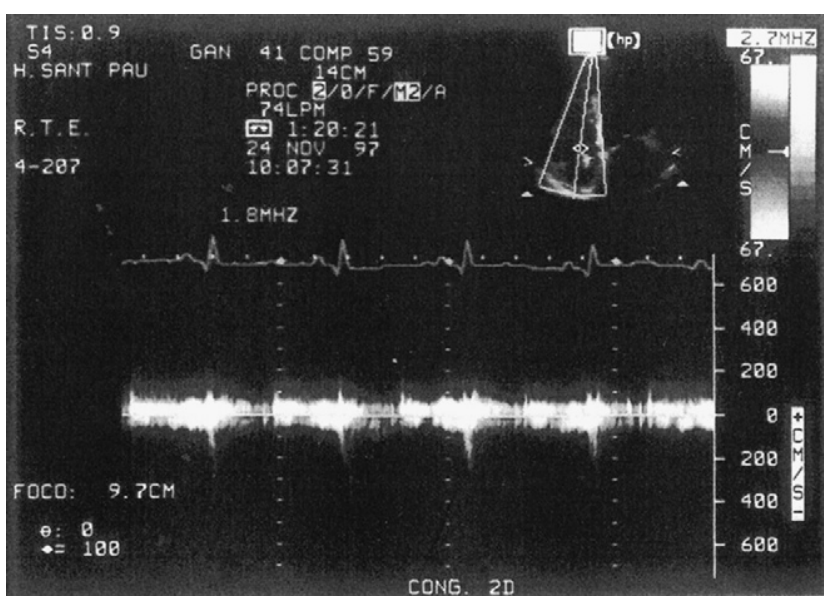

Figura 1 Imagen ecocardiográfica que muestra el patrón número 1 de regurgitación tricúspidea en base a la calidad de la señal; 1 = señal protosistólica que no permite la medida de la velocidad pico.

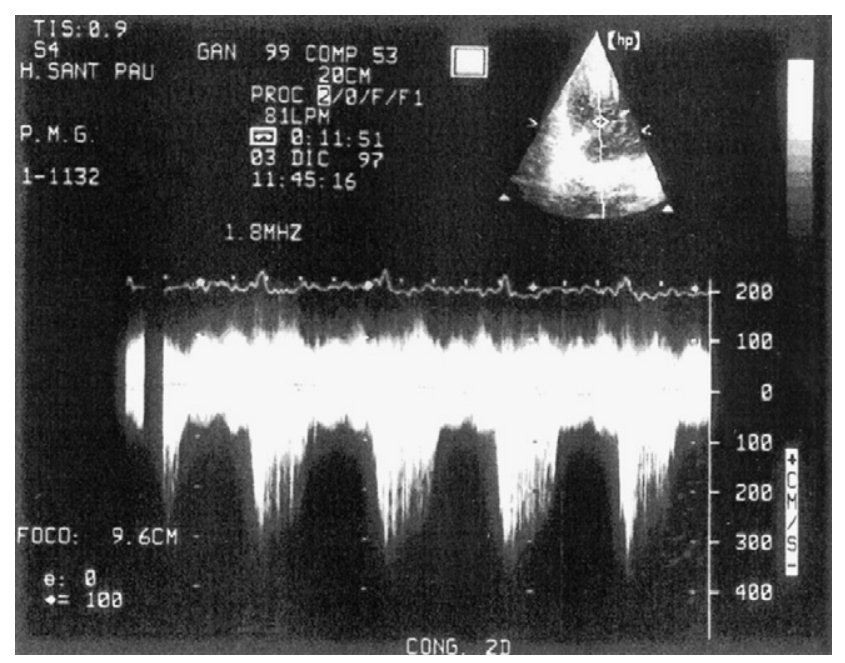

Figura 2 Imagen ecocardiográfica que muestra el patrón número 2 de regurgitación tricúspidea en base a la calidad de la señal; 2 =señal de intensidad no homogénea pero que permite la medida de la velocidad pico.

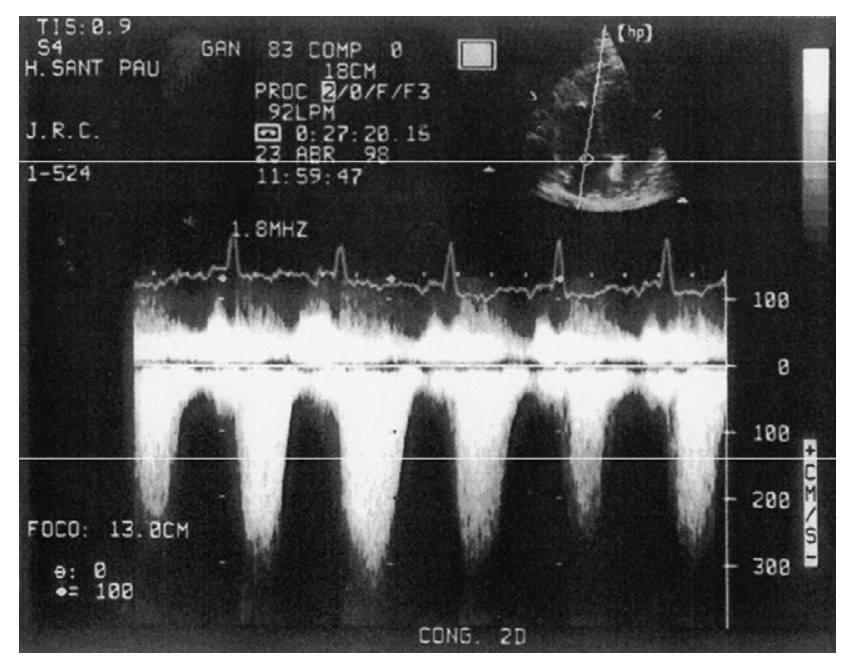

Figura 3 Imagen ecocardiográfica que muestra el patrón número 3 de regurgitación tricúspidea en base a la calidad de la señal; $3=$ señal uniforme y pansistólica.

Angelsen, fue utilizada para el calculo del gradiente sistólico transtricuspideo que equivale a $4 v^{2}$ en el que «V» es la velocidad pico de la curva de flujo de RT en $\mathrm{m} / \mathrm{s}$.

La PAPs fue calculada como sugirieron Currie et $\mathrm{al}^{16}$ por la ecuación siguiente: PAPs $=4 \times$ ( $v$. máxima transtricuspidea) +10 (presión de aurícula derecha). Se eligió $10 \mathrm{mmHg}$ como valor arbitrario de la aurícula derecha. El examen fue considerado técnicamente aceptable ya que la clasificación de la velocidad fue similar por los 3 ecocardiografistas y las medidas y valores variaron menos de un $10 \%$ entre los observadores.

Tras la administración del contraste intravenoso, $2,5 \mathrm{~g}$ de un agente comercializado compuesto de galactosa IEA (intravenous echo-enhancing agent, Levovist, Schering España, Madrid) diluido a una concentración de $200 \mathrm{mg} / \mathrm{ml}$, las medidas fueron repetidas, ajustando la señal para evitar el fenómeno de saturación de la misma. 
Con el objetivo de evitar cualquier sesgo en la medida del valor de la PAPs secundario a la administración del ecocontraste per se, 15 pacientes de la unidad de reanimación cardiovascular fueron incluidos en el estudio. El médico tratante de estos enfermos los había monitorizado con un catéter de Swan-Ganz para optimización del manejo hemodinámico. Los pacientes con insuficiencia renal o cardiaca y los transplantados no fueron incluidos en el estudio. El contraste IEA fue utilizado en la misma dosis que para el estudio de los pacientes EPOC y una media de 3 lecturas fue realizada para cada paciente antes y después de la administración de contraste utilizando el valor de la PAPs obtenido por el catéter Swan-Ganz. La correcta posición del catéter fue realizada con la radiografía de tórax y por la morfología de las curvas de presión de la arteria pulmonar.

\section{Análisis estadístico}

La estadística se realizó usando el programa SPSS (SPSS 13 Chicago, IL). Las variables continuas fueron expresadas como media \pm desviación estándar. Las variables fueron comparadas usando el test de Wilcoxon para test pareados de muestras relacionadas. Un valor de la $\mathrm{p}<0,05$ fue utilizada para mostrar una diferencia significativa.

\section{Resultados}

Cuarenta y dos pacientes EPOC ambulatorios fueron incluidos, 18 mujeres y 24 hombres. La edad media fue de 66 años (rango 39-84). Ningún paciente presentaba patología cardiaca en un examen ecocardiográfico de rutina.

Cuarenta y uno de los 42 pacientes EPOC tenía señal de regurgitación tricuspidea suficientemente clara para clasificarla en 1 de los 4 patrones definidos previamente. Hubo un paciente en el que no se pudo calcular por no existir insuficiencia tricuspidea detectable ecocardiográficamente con y sin contraste. En el estudio basal una señal Doppler de RT fue identificada en 31 de los 41 pacientes con RT (76\%) y la máxima velocidad pudo calcularse en 20 pacientes (49\%) basándose en los patrones 2 y 3 . Diecisiete pacientes fueron clasificados como 2 y 3 como 3 en la escala de la señal de RT en el estudio basal. Tras la inyección de contraste IEA una señal Doppler de RT fue detectada en 41 pacientes (100\%) y la PAPs fue calculada en 39 pacientes (95\%). Dos señales fueron clasificadas como 1,11 como 2 y 28 como 3 en la escala de RT (fig. 4). En los 20 pacientes en los que la PAPs fue estimada antes del contraste, un incremento significativo en la magnitud de la señal de RT fue observada, resultando en velocidades máximas más altas. El valor de la PAPs aumentó significativamente siendo el valor medio de la PAPs $44 \pm 10 \mathrm{mmHg}$ en situación basal vs. $55 \pm 15 \mathrm{~mm} \mathrm{Hg}$ tras el contraste, con una diferencia media de $12,7 \pm 59,5 \mathrm{mmHg}$ (IC 95\%, 8,2-17,2) ( $<<0,0001)$ (fig. 5).

Para descartar la posibilidad de cambios en el valor de la PAPs debido a la administración de contraste per se, la PAPs fue estimada de manera invasiva en 15 pacientes que estaban monitorizados con un catéter de Swan-Ganz en la unidad de cirugía cardiaca. Ningún cambio ocurrió en el valor de la PAPs medida por Swan-Ganz tras la administración de contraste $35 \pm 10 \mathrm{mmHg}$ antes del contraste vs. $35 \pm 9 \mathrm{mmHg}$ tras el mismo, $p=0,69$ (fig. 6).

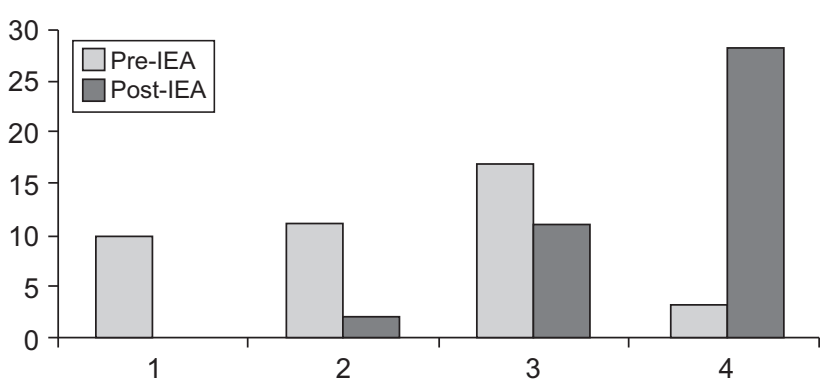

Figura 4 Histograma que muestra el número de pacientes antes (gris) y después de la administración de contraste clasificados en uno de los 4 patrones de regurgitación tricúspidea $(\mathrm{RT})$; $0=$ ausencia de regurgitación; $1=$ señal protosistólica que no permite la medida de la velocidad pico; $2=$ señal de intensidad no homogénea pero que permite la medida de la velocidad pico y $3=$ señal uniforme y pansistólica.

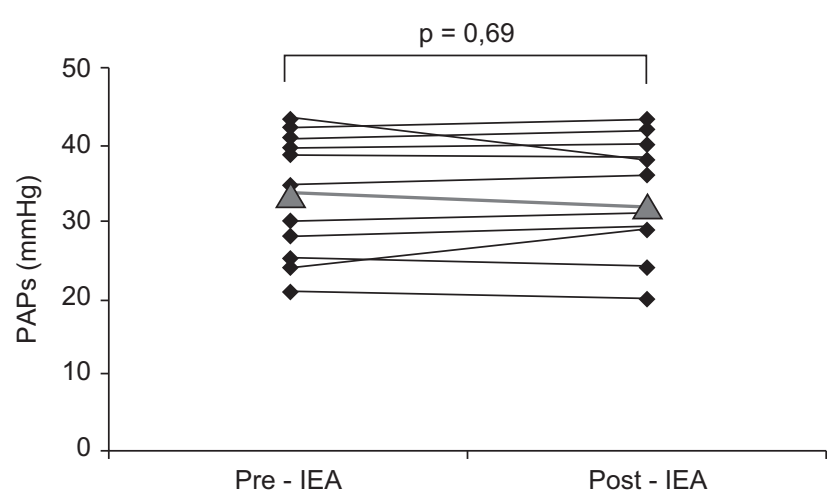

Figura 5 Figura que muestra los valores individuales de la presión de arteria pulmonar sistólica (PAPs) medida por el catéter de Swan-Ganz, en $\mathrm{mmHg}$, en los 15 pacientes de la unidad de cirugía cardiaca antes (izquierda) y tras la administración de contraste IEA (derecha). Ninguna diferencia significativa fue observada en el valor de la PAPs antes o después de la administración de contraste.

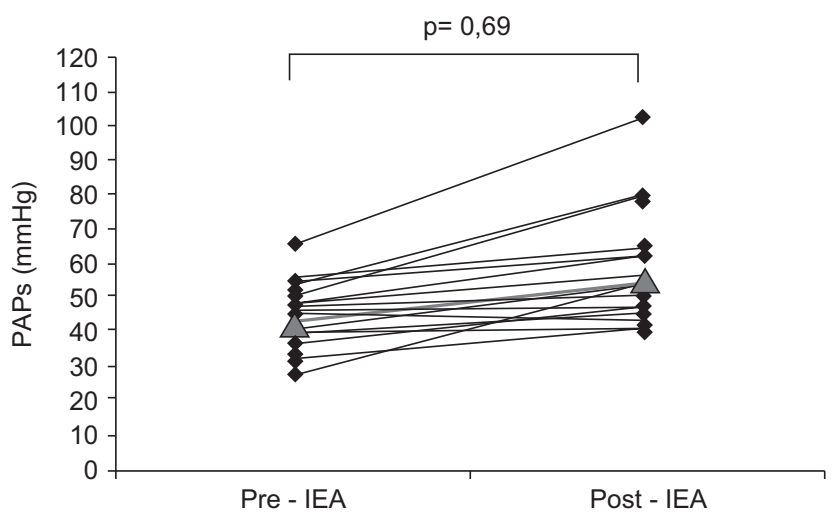

Figura 6 Figura que muestra los valores individuales de la presión de arteria pulmonar sistólica en $\mathrm{mm} \mathrm{Hg}$, medida por ecocardiografía Doppler en los 20 pacientes EPOC antes (izquierda) y después (derecha) del contraste. Un incremento significativo fue observado en la PAPs tras la administración del contraste IEA, $\mathrm{p}<0,01$. 


\section{Discusión}

En este estudio realizado en pacientes estables EPOC hemos encontrado que el uso de un contraste ecocardiográfico intravenoso incrementa el número de pacientes en los que la PAPs puede ser estimado de manera no invasiva. También hemos encontrado que el uso de este contraste puede ser útil para estimar el valor de la PAPs, evitando una infra o supraestimación secundarias a las dificultades en la obtención de una señal real en estos pacientes.

\section{Agente de contraste ecocardiográfico}

La eficacia del suero salino agitado para obtener una señal óptima de Doppler en pacientes EPOC ha sido descrita en estudios previos ${ }^{13,14}$. Contrariamente al suero salino agitado, Levovist ${ }^{\mathbb{B}}$ es un contraste transpulmonar que también influye en el Doppler color y espectral de las señales de las cavidades cardiacas izquierdas, permitiendo por tanto una mejor evaluación en la estenosis aórtica, insuficiencia mitral y en el flujo de las venas pulmonares ${ }^{17}$. Un agente que refuerza el patrón de flujo de las cavidades derechas ofrece varias posibilidades. Si bien el suero salino agitado no puede ser administrado por una inyección continua, Levovist ${ }^{\circledR}$ puede administrarse lentamente potenciando la señal tanto en las cavidades derechas como izquierdas. La señal obtenida por el suero salino agitado también contiene más artefactos y precisa de varias dosis para obtener imágenes interpretables. Existen en la literatura estudios que han demostrado como el uso de contraste puede ayudar en la medida de la presión de arteria pulmonar sistólica por la señal de insuficiencia tricuspidea ${ }^{10,11}$.

\section{Agentes de contraste intravenosos compuestos de galactosa}

En el presente estudio hemos podido demostrar que el aumento de las propiedades eco reflectantes de la sangre obtenidas por el agente intravenoso basado en galactosa (Levovist ${ }^{\circledR}$ facilita la detección de una señal Doppler fiable de regurgitación tricuspidea en estos pacientes. Si bien la mejora en la calidad de la señal confirma nuestra hipótesis, un incremento en el valor de la velocidad máxima y por tanto en la optimización para el cálculo del valor de la PAPs fue un hallazgo casual. Aún así se han descrito hallazgos similares en resultados de estudios coronarios utilizando el mismo agente de contraste IEA, donde las velocidades máximas tras la inyección del contraste aumentaron ${ }^{18}$. Señales Doppler de baja intensidad, no analizadas por el equipo por considerarlas ruido, son integradas en la velocidad tras la inyección de contraste (fig. 7). Estas señales de baja intensidad, cuando son incorporadas, evitan una infraestimación de las velocidades máximas y por tanto del valor de la PAPs. Una infraestimación sistemática en pacientes con hipertensión pulmonar severa, previamente descrita, puede resultar en la exclusión de las señales de baja intensidad por el aparato ${ }^{19,20}$.

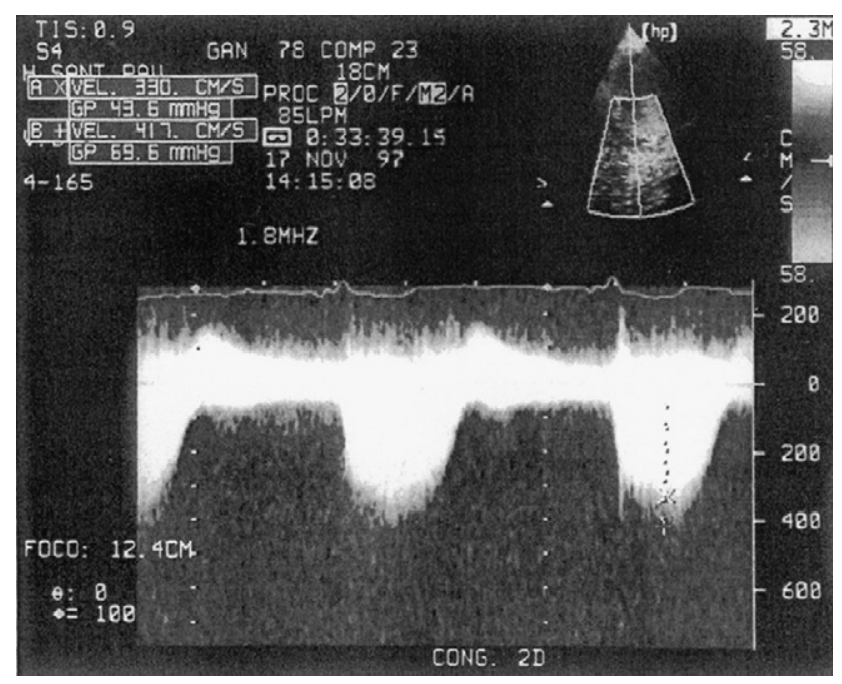

Figura 7 Señal de Doppler que tras la administración de contraste IEA muestra un doble contorno. El contorno interior es de alta intensidad con una velocidad pico de de $3,3 \mathrm{~m} / \mathrm{s}$, el exterior tiene una menor intensidad y una velocidad pico mayor, de $4,17 \mathrm{~m} / \mathrm{s}$.

\section{Estimación del valor de la presión sistólica de arteria pulmonar}

Las mediciones realizadas en los 15 pacientes de la unidad de cirugía cardiaca monitorizados con catéter de Swan-Ganz nos facilitaron saber si el uso de contraste IEA per se podía aumentar el valor de la PAPs. No hubo diferencias en los valores pre y post administración del IEA, sugiriendo por tanto la ausencia de incremento relativo a la utilización de este agente (fig. 6). El uso de un método independiente en estos pacientes de cirugía cardiaca nos ayudó a perfilar esta conclusión. Aún así, no podemos descartar totalmente si el efecto del contraste IEA en pacientes EPOC (incrementando la velocidad de RT), pudo ser secundario a una sola respuesta. En un estudio realizado en perros donde utilizaron el Levovist ${ }^{\circledR}$, se observó un incremento significativo en el valor de la PAPs y se observó aumento de la resistencia vascular pulmonar en los perros cuando se incrementaba la dosis del contraste IEA (cuando esta dosis era equivalente a 7 veces más que la recomendada en humanos). A dosis más bajas (aquellas cuyo valor era el recomendado para humanos), un mínimo incremento significativo se observó en el valor de la PAPs ${ }^{21}$. Schwarz et al atribuyeron este hallazgo al posible efecto vasoconstrictor del agente de contraste. Por tanto, no se puede descartar de manera categórica que algún efecto en la vascularización pulmonar de los pacientes EPOC podría haber contribuido a una respuesta vasoconstrictora tras la administración de IEA.

\section{Valor de la presión sistólica de arteria pulmonar}

El valor de la PAPs encontrada en el presente estudio se encuentra en concordancia con otros valores descritos en la literatura en pacientes EPOC. La mayoría de ellos desarrolla de ligera a moderada hipertensión arterial pulmonar ${ }^{9,22,23}$. En el estudio de Scharf et $\mathrm{al}^{23}$ el mayor porcentaje de 
pacientes EPOC ambulatorios tenían un valor de PAPs entre $30-45 \mathrm{~mm} \mathrm{Hg}$, medida por cateterismo cardiaco derecho. En otro estudio la presión de arteria pulmonar sistólica fue de $43 \pm 10 \mathrm{~mm} \mathrm{Hg}$, en este caso medida por ecocardiografia en pacientes EPOC estables ${ }^{9}$. La pequeña diferencia entre nuestro valores y los descritos en estos 2 estudios $^{9,23}$ citados podría deberse a que nosotros mostramos los valores encontrados en 20 pacientes en los que el valor de PAPs fue posible obtener sin contraste. Esto podría haber creado un sesgo en la selección de pacientes, teniendo en cuenta principalmente a pacientes con valores más elevados de PAPs obtenidos por ecocardiografia.

\section{Implicaciones clínicas}

El desarrollo de hipertensión pulmonar ligera a moderada en pacientes EPOC conlleva un pronóstico infausto ${ }^{3}$. Estos pacientes han de identificarse ya que son candidatos a terapias específicas dirigidas a reducir la resistencia vascular pulmonar. El diagnóstico de hipertensión pulmonar severa en estos pacientes es poco frecuente ${ }^{23-25}$ y obliga a descartar otras potenciales causas de hipertensión pulmonar como el síndrome de apnea del sueño, el síndrome hipoventilación obesidad o la hipertensión pulmonar idiopática $^{25}$. Además, los pacientes EPOC suelen presentar comorbilidad asociada al tabaquismo, como la miocardiopatía isquémica, una patología que hace emerger la hipertensión pulmonar y consecuentemente el fallo cardia$\mathrm{Co}^{26}$. Estas potenciales comorbilidades pueden contribuir de manera dramática al pronóstico de estos pacientes. Así como la PAPs puede ser un estimador indirecto del efecto de la hipoxia en la vasculatura pulmonar en los pacientes EPOC, su cálculo es de vital importancia para la evaluación, el tratamiento y el control evolutivo de estos pacientes.

\section{Limitaciones}

En nuestro estudio no hemos clasificado la severidad de los EPOC, y esto podría haber sido interesante para realizar correlaciones entre la severidad del intercambio gaseoso, el grado de obstrucción al flujo aéreo, el índice de masa muscular, la disnea o la tolerancia al ejercicio ${ }^{27}$ con el valor de la PAPs. El presente estudio no fue diseñado para responder a esta pregunta.

Otra limitación del estudio es que se eligió $10 \mathrm{~mm} \mathrm{Hg}$ como valor arbitrario de la aurícula derecha. Hay estudios que han utilizado este valor como una medida aceptable del cálculo de presión de la aurícula derecha, si bien lo más adecuado hubiera sido la estimación a partir del diámetro de la vena cava inferior determinado por modo $M$ ecográfico, técnica que se puede realizar sin gran dificultad en pacientes de cuidados intensivos ${ }^{28}$.

En nuestro estudio hemos utilizado solo el Doppler como método de evaluación del valor de la PAPs en pacientes EPOC. Otros métodos incluyen la velocidad de regurgitación pulmonar, el perfil de la velocidad del flujo pulmonar y el tiempo de relajación isovolumétrico del ventrículo derecho $^{8,29}$. Aún así el método que hemos utilizado en este estudio es el más utilizado y también el mejor correlacionado con la medida de la PAPs realizado de manera invasiva. Hemos utilizado solo el eje 4 cámaras para la medida de velocidad de RT. Aunque las imágenes del ángulo subcostal pueden proporcionar imágenes óptimas, la estimación de la velocidad de RT puede ser más difícil de obtener, siendo a veces imposible.

En el presente estudio no se realizó simultáneamente ecocardiografia y cateterización cardiaca derecha en los 15 pacientes de la unidad cardiovascular porque las diferencias derivadas de las diferentes posturas del paciente para la medida óptima con cada método podrían haber invalidado la correlación de valores. Además, una cateterización derecha suele magnificar la RT ya que el catéter atraviesa la válvula.

\section{Conclusión}

La administración de contraste basado en galactosa (Levovist ${ }^{\circledR}$ ) en pacientes EPOC puede aumentar el número de pacientes en los que la presión sistólica de la arteria pulmonar puede ser calculada de manera precisa evitando una infraestimación.

La administración de contraste puede así mismo evitar o disminuir la variabilidad inter e intra ecocardiografistas, lo que en determinadas ocasiones puede limitar la comparación entre valores.

En pacientes con difícil ventana ecocardiográfica tales como los EPOC o los pacientes ventilados en $\mathrm{UCl}$ la administración de contraste puede optimizar el cálculo de la PAPs.

\section{Bibliografía}

1. MacNee W. Pathophysiology of cor pulmonale in chronic obstructive pulmonary disease. Part two. Am J Respir Crit Care Med. 1994;150:1158-68.

2. Kessler RM, Faller G, Fourgaut B, Mennecier E, Weitzenblum. Predictive factors of hospitalization for acute exacerbation in a series of 64 patients with chronic obstructive pulmonary disease. Am J Respir Crit Care Med. 1999;59:158-64.

3. Keller CA, Shepard Jr JW, Chun DS, Vasquez P, Dolan GF. Pulmonary hypertension in chronic obstructive pulmonary disease. Multivariate analysis. Chest. 1986;90:185-92.

4. Oswald-Mammosser M, Weitzenblum E, Quoix E, Moser G, Chaouat A, Charpentier C, et al. Prognostic factors in COPD patients receiving long-term oxygen therapy. Importance of pulmonary artery pressure. Chest. 1995;107:1193-8.

5. Continuous or nocturnal oxygen therapy in hypoxemic chronic obstructive lung disease: a clinical trial. Nocturnal Oxygen Therapy Trial Group. Ann Intern Med. 1980;93:391-8.

6. Long term domiciliary oxygen therapy in chronic hypoxic cor pulmonale complicating chronic bronchitis and emphysema. Report of the Medical Research Council Working Party. Lancet. 1981;1:681-6.

7. Weitzenblum E, Sautegeau A, Ehrhart M, Mammosser $M$, Pelletier A. Long-term oxygen therapy can reverse the progression of pulmonary hypertension in patients with chronic obstructive pulmonary disease. Am Rev Respir Dis. 1985;131:493-8.

8. Tramarin R, Torbicki A, Marchandise B, Laaban JP, Morpurgo M. Doppler echocardiographic evaluation of pulmonary artery pressure in chronic obstructive pulmonary disease. A European multicentre study. Working Group on Noninvasive Evaluation of Pulmonary Artery Pressure. European Office of the World Health Organization, Copenhagen. Eur Heart J. 1991;12:103-11.

9. Boussuges A, Pinet C, Molenat F, Burnet $\mathrm{H}$, Ambrosi P, Badier M, et al. Left atrial and ventricular filling in chronic obstructive 
pulmonary disease. An echocardiographic and Doppler study. Am J Respir Crit Care Med. 2000;162:670-5.

10. Tan HC, Fung KC, Kritharides L. Agitated colloid is superior to saline and equivalent to levovist in enhancing tricuspid regurgitation Doppler envelope and in the opacification of right heart chambers: a quantitative, qualitative, and cost-effectiveness study. J Am Soc Echocardiogr. 2002;15:309-15.

11. Milan A, Magnino C, Veglio F. Echocardiographic indexes for the non-invasive evaluation of pulmonary hemodynamics. J Am Soc Echocardiogr. 23:225-39.

12. Dini FL, Nuti R, Barsotti L, Baldini U, Dell’Anna R, Micheli G. Doppler-derived mitral and pulmonary venous flow variables are predictors of pulmonary hypertension in dilated cardiomyopathy. Echocardiography. 2002;19:457-65.

13. Himelman RB, Struve SN, Brown JK, Namnum P, Schiller NB. Improved recognition of cor pulmonale in patients with severe chronic obstructive pulmonary disease. Am J Med. 1988;84:891-8.

14. Himelman RB, Stulbarg M, Kircher B, Lee E, Kee L, Dean NC, et al. Noninvasive evaluation of pulmonary artery pressure during exercise by saline-enhanced Doppler echocardiography in chronic pulmonary disease. Circulation. 1989;79:863-71.

15. Carreras F, Borras X, Auge JM, Pons-Llado G. Pulsed Doppler assessment of tricuspid regurgitation: usefulness of regurgitant signal patterns for estimation of severity. Angiology. 1988;39: 788-94.

16. Currie PJ, Seward JB, Chan KL, Fyfe DA, Hagler DJ, Mair DD, et al. Continuous wave Doppler determination of right ventricular pressure: a simultaneous Doppler-catheterization study in 127 patients. J Am Coll Cardiol. 1985;6:750-6.

17. Von Bibra H, Sutherland G, Becher H, Neudert J, Nihoyannopoulos P. Clinical evaluation of left heart Doppler contrast enhancement by a saccharide-based transpulmonary contrast agent. The Levovist Cardiac Working Group. J Am Coll Cardiol. 1995;25:500-8.

18. Kozakova M, Palombo C, Zanchi M, Distante A, L'Abbate A. Increased sensitivity of flow detection in the left coronary artery by transesophageal echocardiography after intravenous administration of transpulmonary stable echocontrast agent. J Am Soc Echocardiogr. 1994;7:327-36.
19. Brecker SJ, Gibbs JS, Fox KM, Yacoub MH, Gibson DG. Comparison of Doppler derived haemodynamic variables and simultaneous high fidelity pressure measurements in severe pulmonary hypertension. Br Heart J. 1994;72:384-9.

20. Chan KL, Currie PJ, Seward JB, Hagler DJ, Mair DD, Tajik AJ. Comparison of three Doppler ultrasound methods in the prediction of pulmonary artery pressure. J Am Coll Cardiol. 1987;9:549-54.

21. Schwarz KQ, Bezante GP, Chen X, Phillips D, Schlief R. Hemodynamic effects of microbubble echo contrast. J Am Soc Echocardiogr. 1996;9:795-804.

22. Thabut G, Dauriat G, Stern JB, Logeart D, Levy A, MarrashChahla R, et al. Pulmonary hemodynamics in advanced COPD candidates for lung volume reduction surgery or lung transplantation. Chest. 2005;127:1531-6.

23. Scharf SM, lqbal M, Keller C, Criner G, Lee S, Fessler HE. Hemodynamic characterization of patients with severe emphysema. Am J Respir Crit Care Med. 2002;166:314-22.

24. Stevens D, Sharma K, Szidon P, Rich S, McLaughlin V, Kesten S. Severe pulmonary hypertension associated with COPD. Ann Transplant. 2000;5:8-12.

25. Chaouat A, Bugnet AS, Kadaoui N, Schott R, Enache I, Ducolone A, et al. Severe pulmonary hypertension and chronic obstructive pulmonary disease. Am J Respir Crit Care Med. 2005;172: 189-94.

26. Rabe KF, Hurd S, Anzueto A, Barnes PJ, Buist SA, Calverley P, et al. Global Strategy for the Diagnosis, Management, and Prevention of COPD-2006 Update. Am J Respir Crit Care Med. 2007.

27. Celli BR, Cote CG, Marin JM, Casanova C, Montes de Oca M, Mendez RA, et al. The body-mass index, airflow obstruction, dyspnea, and exercise capacity index in chronic obstructive pulmonary disease. N Engl J Med. 2004;350:1005-12.

28. Garcia-Vicente E, Campos-Nogue A, Gobernado Serrano MM. Echocardiography in the Intensive Care Unit. Med Intensiva. 2008;32:236-47.

29. Oh JK, Seward JB, Tajik AJ. The Echo Manual, 2nd ed. Lippincott-Raven Publishers; 1998 p. 187-90. 